\title{
BOREL AND ANALYTIC ONE-ONE PARAMETRIZATIONS OF THE COUNTABLE SETS OF REALS
}

\author{
HOWARD BECKER
}

(Communicated by Thomas J. Jech)

\begin{abstract}
We consider whether or not the parametrizations of the title exist. In the Borel case, they do not exist. In the analytic case, this is independent of ZFC.
\end{abstract}

R. M. Shortt asked the following two questions [3, p. 320]. "Let $\mathbf{C}$ be the class of all countable subsets of a Polish space $X$. Can $\mathbf{C}$ be given a one-one parametrization $a \mapsto C_{a}$ by an analytic set $A$ in such a way that the membership relation $\left\{(a, x): x \in C_{a}\right\}$ is Borel (analytic) in $A \times X$ ?"

The purpose of this paper is to answer these questions. For Borel, the answer is no-no such parametrization exists. For analytic, the question cannot be decided in ZFC. If every PCA $\left(\Sigma_{2}^{1}\right)$ set of reals is Lebesgue measurable then the answer is no and if $V=L$ then the answer is yes.

Definitions. A Polish space is a separable complete metric space. An analytic set (or $\Sigma_{1}^{1}$ set) is the continuous image of a Borel set. If $R$ is a binary relation, then $R_{x}=\{y:(x, y) \in R\}$. A parametrization will always mean a one-to-one parametrization of the class of countable subsets of some fixed uncountable Polish space, $X$, that is, a pair $(A, P)$ such that:

(1) $A \subset X$.

(2) $P \subset A \times X$.

(3) For all $x \in A, P_{x}$ is countable.

(4) For all countable $D \subset X$, there is an $X \in A$ such that $D=P_{x}$.

(5) For all $x, y \in A$, if $x \neq y$ then $P_{x} \neq P_{y}$.

A Borel parametrization is a parametrization in which $A$ is an analytic set and $P$ is a Borel set in $A \times X$, i.e., $P=P^{\prime} \cap(A \times X)$, where $P^{\prime} \subset X^{2}$ is Borel in the Polish space $X^{2}$. An analytic parametrization is one in which both $A$ and $P$ are analytic sets.

All uncountable Polish spaces are Borel isomorphic $[\mathbf{4}, 1 \mathrm{G}]$, so neither of Shortt's questions can depend on what the space $X$ is. (For countable Polish spaces, the problem trivializes.)

\section{Theorems}

THEOREM 1. There does not exist a Borel parametrization.

Received by the editors June 30, 1987.

1980 Mathematics Subject Classification (1985 Revision). Primary 03E15; Secondary 03E35, 04A 15, 28A05.

Research partially supported by NSF Grant DMS-8601731. 
THEOREM 2. If every PCA set of reals is Lebesgue measurable, then there does not exist an analytic parametrization.

THEOREM 3. If $V=L$ then there exists an analytic parametrization.

Remarks. 1. Shortt has informed me that Theorem 1 was proved independently by Blackwell.

2. Each of the two propositions, $V=L$ and "Every PCA set is measurable", is known to be relatively consistent with ZFC, hence so are the conclusions of Theorems 2 and 3. Therefore, Shortt's second question is independent of ZFC. Large cardinal axioms imply that it is true that PCA sets are measurable, hence they imply a negative answer to Shortt's second question. For more information on these matters, see Jech [1] or Moschovakis [4].

3. It can be shown that if $V=L$ then there exists a parametrization in which $A$ is coanalytic $\left(\Pi_{1}^{1}\right)$ and $P$ is Borel in $A \times X$. If PCA sets are measurable then no such parametrization exists.

Proofs. The proofs involve effective descriptive set theory, that is, recursiontheoretic methods. Moschovakis [4] is the canonical reference for this, and we use his notation and terminology.

PROOF OF THEOREM 1 . We work with the space of real numbers. Let $E$ be the equivalence relation: Two reals are equivalent iff their difference is rational. A set $V \subset \mathbf{R}$ is a Vitali set if it contains exactly one element from each $E$-equivalence class. A Vitali set is not Lebesgue measurable. Let $\boldsymbol{\Lambda}$ be the $\sigma$-algebra generated by the $\Pi_{1}^{1}$ sets. Every set in $\boldsymbol{\Lambda}$ is measurable, hence no Vitali set is in $\boldsymbol{\Lambda}$.

Assume there exists a Borel parametrization $(A, P)$; we show that there exists a Vitali set in $\boldsymbol{\Lambda}$. By the von Neumann Selection Theorem [4, 4E.9], $P$ can be uniformized by a $\mathbf{\Lambda}$ set $P^{*}$. Let $f: \mathbf{R} \rightarrow \mathbf{R}$ be the function:

$$
f(w)=\text { the unique real } x \text { such that } x \in A \text { and } P_{x} \text { is } E_{w} .
$$

Claim. $f$ is a Borel-measurable function.

Let $V=\left\{y \in \mathbf{R}:(f(y), y) \in P^{*}\right\}$. Clearly $V$ is a Vitali set. By the claim, $V$ is the preimage of a $\boldsymbol{\Lambda}$ set by a Borel function, hence a $\boldsymbol{\Lambda}$ set. So all that remains to be proved is the claim.

It will suffice to prove that the graph of $f$ is a $\Sigma_{1}^{1}$ set in $\mathbf{R}^{2}$. Let $C=\{(w, x): x \in$ $A$ and $E_{w} \subset P_{x}$ \}. Call a set $D \subset \mathbf{R}^{2}$ good if for every $x \in A$ :

$$
\forall w\left[(w, x) \in D \text { iff } P_{x} \subset E_{w}\right] .
$$

For any good $D, \operatorname{graph}(f)=C \cap D$, and $C$ is clearly $\Sigma_{1}^{1}$. So we need only show that there is a good set $D$ which is $\Sigma_{1}^{1}$. Let $P^{\prime} \subset \mathbf{R}^{2}$ be Borel such that $P=P^{\prime} \cap(A \times \mathbf{R})$. Let $z \in \omega^{\omega}$ be such that $P^{\prime}$ is $\Sigma_{1}^{1}(z)$. By the Effective Perfect Set Theorem $[4,4 \mathrm{~F} .1]$, for all $x \in A$, every element of $P_{x}$ is $\Delta_{1}^{1}(x, z)$. So the set

$$
D=\left\{(w, x):\left(\forall y \in \Delta_{1}^{1}(x, z)\right)\left(\text { if } y \in P_{x}^{\prime} \text { then } y \in E_{w}\right)\right\}
$$

is good. By a theorem of Kleene (see [4, 4D.3]), the pointclass $\Sigma_{1}^{1}$ is closed under quantification of the form $\forall u \in \Delta_{1}^{1}(v)$; so $D$ is $\Sigma_{1}^{1}$.

The referee has called my attention to the theorem of Luzin (see [4, 4F.17]) which states that given a $\Sigma_{1}^{1}$ set $A$, and a set $P \subset A \times \mathbf{R}$ which is Borel in $A \times \mathbf{R}$ and 
has countable sections, there is a sequence $\left\langle P^{n}\right\rangle_{n \in \omega}$ of sets, each Borel in $A \times \mathbf{R}$, such that $P=\bigcup_{n} P^{n}$ and such that for all $x \in A$ and all $n \in \omega$ :

There is a $y \in P_{x} \Leftrightarrow$ There is exactly one $y$ such that $y \in P_{x}^{n}$.

Using this theorem, rather than the Effective Perfect Set Theorem, one can eliminate the recursion theory from the above proof of Theorem 1. Luzin's Theorem can also be used in place of the von Neumann Selection Theorem-it gives a Borel $P^{*}$.

PROOF OF THEOREM 2. The proof is similar to that of Theorem 1. The function $f$ is no longer Borel; but it is $\Delta_{2}^{1}$, so the Vitali set is $\Sigma_{2}^{1}$.

We now assume the axiom $V=L$. A knowledge of the basic facts about $L$ is needed to follow the proof of Theorem 3. This topic is covered in Jech [1] and Moschovakis [4]. We work with Baire space, $\omega^{\omega}$. Thus $\leq_{L}$ is a wellordering of the universe, and $\leq_{L} \uparrow \omega^{\omega}$ has order-type $\aleph_{1}$. Let $\omega_{1}^{x}$ be the least ordinal not recursive in $x\left(x \in \omega^{\omega}\right)$. Let

$$
\mathscr{C}_{1}=\left\{x \in \omega^{\omega}: x \in L_{\alpha} \text { for some } \alpha<\omega_{1}^{x}\right\} .
$$

Then $\mathscr{C}_{1}$ is a $\Pi_{1}^{1}$ set, every element of $\omega^{\omega}$ is recursive in some element of $\mathscr{C}_{1}$, and for any $x \in \mathscr{C}_{1}$, one can talk about the part of the universe that is $\leq_{L} x$ in a $\Sigma_{1}^{1}(x)$ way, and also in a $\Pi_{1}^{1}(x)$ way, uniformly in $x$. For more information about $\mathscr{C}_{1}$, see Kechris [2].

PROOF OF THEOREM 3. It will suffice to show that there is a $P \subset \omega^{\omega} \times \omega^{\omega}$ such that:

(1) For all $x \in \omega^{\omega}, P_{x}$ is countable.

(2) For all countable $D \subset \omega^{\omega}$, there is an $x \in \omega^{\omega}$ such that $D=P_{x}$.

(3) For all $w, x \in \omega^{\omega}$, if $w \neq x$ then either $P_{w} \neq P_{x}$ or else $P_{w}=P_{x}=\varnothing$.

(4) $P$ is $\Sigma_{1}^{1}$.

The definition of $P$ is as follows.

(a) If $x \notin \mathscr{C}_{1}$, then $P_{x}=\left\{y: y<_{T} x\right\} \cup\{x\}$, where $<_{T}$ denotes strict Turing reducibility.

(b) If $x \in \mathscr{C}_{1}$, let $P_{x}$ be the $\leq_{L}$-least set $D \subset \omega^{\omega}$ satisfying the following three conditions, if such a $D$ exists; if no such $D$ exists, then $P_{x}=\varnothing$.

(i) $D$ is countable; moreover, there is an ordinal $\alpha$ such that an enumeration of $D$ occurs in $L_{\alpha}$, but $x \notin L_{\alpha}$.

(ii) For all $y \in D, y<_{T} x$.

(iii) For all $w \in \omega^{\omega}$, if $w<_{L} x$ then $D \neq P_{w}$. (Here $w$ may or may not be in $\mathscr{C}_{1}$.)

Clearly $P$ satisfies (1). It follows from the facts that $\leq_{L} \mid \mathscr{C}_{1}$ and $\leq_{L} \mid\{D \subset$ $\omega^{\omega}: D$ countable $\}$ have order-type $\aleph_{1}$, and that for every $z \in \omega^{\omega}$, there is an $x \in \mathscr{C}_{1}$ with $z \leq_{T} x$, that $P$ satisfies (2). To prove (3), suppose that $w<_{L} x$; we show that either $P_{w} \neq P_{x}$ or else both are empty. If $x \in \mathscr{C}_{1}$, this follows from (b) (iii). So assume $x \notin \mathscr{C}_{1}$. If $w \in \mathscr{C}_{1}$, then it follows from (b) (i) and the transitivity of the $L_{\alpha}$ 's that all members of $P_{w}$ are $\leq_{L} w$; since $w<_{L} x, x \notin P_{w}$; by (a), $x \in P_{x}$; hence $P_{w} \neq P_{x}$. So assume that neither $w$ nor $x$ is in $\mathscr{C}_{1}$. If $P_{w}=P_{x}$, then $w<_{T} x$ and $x<_{T} w$, which is absurd.

All that remains to be proved is that $P$ is $\Sigma_{1}^{1}$. Let $\widehat{P}=P \cup\left\{(x, y): x \notin \mathscr{C}_{1}\right\}$. Note that

$$
P=\left(\widehat{P} \cap\left\{(x, y): y<_{T} x\right\}\right) \cup\left\{(x, y): x \notin \mathscr{C}_{1} \text { and } y=x\right\}
$$


So it will suffice to show that $\widehat{P}$ is $\Sigma_{1}^{1}$. The following is a $\Sigma_{1}^{1}$-definition of $\widehat{P}$ :

$(x, y) \in \widehat{P} \Leftrightarrow\left(\forall z \in \Delta_{1}^{1}(x)\right)$ [if $z$ encodes a countable ordinal $\alpha$ and $x \in L_{\alpha}$ then $L_{\alpha} \vDash$ " $y \in P_{x}$ "].

\section{REFERENCES}

1. T. J. Jech, Set theory, Academic Press, New York, 1978.

2. A. S. Kechris, The theory of countable analytical sets, Trans. Amer. Math. Soc. 202 (1975), 259-297.

3. D. Kölzow and D. Maharam-Stone, Eds., Measure theory, Oberwolfach 1983, Lecture Notes in Math., vol. 1089, Springer-Verlag, Berlin and New York, 1984.

4. Y. N. Moschovakis, Descriptive set theory, North-Holland, Amsterdam, 1980.

Department of Mathematics, University of South Carolina, Columbia, SOUTH CAROLINA 29208 